\title{
Safety and efficacy of landiolol hydrochloride for prevention of atrial fibrillation after cardiac surgery in patients with left ventricular dysfunction: Prevention of Atrial Fibrillation After Cardiac Surgery With Landiolol Hydrochloride for Left Ventricular Dysfunction (PLATON) trial
}

\author{
Akira Sezai, MD, PhD, Shunji Osaka, MD, PhD, Hiroko Yaoita, MD, Yusuke Ishii, MD, \\ Munehito Arimoto, MD, Hiroaki Hata, MD, PhD, and Motomi Shiono, MD, PhD
}

\begin{abstract}
Objectives: We previously conducted a prospective study of landiolol hydrochloride (INN landiolol), an ultrashort-acting $\beta$-blocker, and reported that it could prevent atrial fibrillation after cardiac surgery. This trial was performed to investigate the safety and efficacy of landiolol hydrochloride in patients with left ventricular dysfunction undergoing cardiac surgery.

Methods: Sixty patients with a preoperative left ventricular ejection fraction of less than $35 \%$ were randomly assigned to 2 groups before cardiac surgery and then received intravenous infusion with landiolol hydrochloride (landiolol group) or without landiolol (control group). The primary end point was occurrence of atrial fibrillation as much as 1 week postoperatively. The secondary end points were blood pressure, heart rate, intensive care unit and hospital stays, ventilation time, ejection fraction, biomarkers of ischemia, and brain natriuretic peptide.
\end{abstract}

Results: Atrial fibrillation occurred in 3 patients (10\%) in the landiolol group versus $12(40 \%)$ in the control group, and its frequency was significantly lower in the landiolol group $(P=.002)$. During the early postoperative period, levels of brain natriuretic peptide and ischemic biomarkers were significantly lower in the landiolol group than the control group. The landiolol group also had a significantly shorter hospital stay $(P=.019)$. Intravenous infusion was not discontinued for hypotension or bradycardia in either group.

Conclusions: Low-dose infusion of landiolol hydrochloride prevented atrial fibrillation after cardiac surgery in patients with cardiac dysfunction and was safe, with no effect on blood pressure. This intravenous $\beta$-blocker seems useful for perioperative management of cardiac surgical patients with left ventricular dysfunction. (J Thorac Cardiovasc Surg 2015;150:957-64)

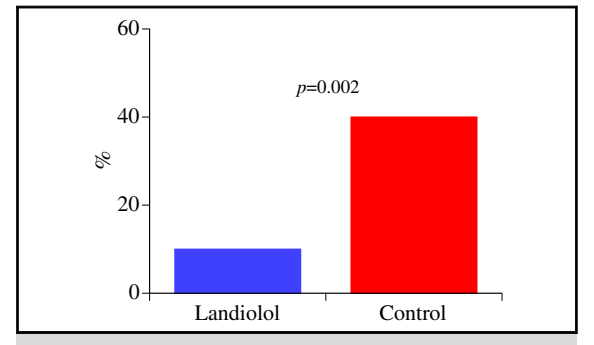

Postoperative atrial fibrillation was significantly lower in the landiolol group.

Central Message

Landiolol hydrochloride seems to be useful for perioperative management of cardiac surgical patients with left ventricular dysfunction.

\section{Perspective}

Low-dose infusion of landiolol hydrochloride prevented atrial fibrillation after cardiac surgery in patients with cardiac dysfunction and was safe, with no effect on their blood pressure.

See Editorial Commentary page 965.

\footnotetext{
From the Department of Cardiovascular Surgery, Nihon University School of Medicine, Tokyo, Japan.

Supported by a grant for scientific research from the Japanese Ministry of Education, Culture, Sports, Science, and Technology (No. 24592075).

Clinical trial registration information: UMIN (http://www.umin.ac.jp/), study ID: UMIN000002160.

Received for publication Dec 24, 2014; revisions received June 29, 2015; accepted for publication July 4, 2015; available ahead of print Aug 5, 2015.

Address for reprints: Akira Sezai, MD, PhD, Department of Cardiovascular Surgery,

Nihon University School of Medicine, 30-1 Oyaguchi-kamimachi Itabashi-ku Tokyo 173-8610, Japan (E-mail: asezai.med@gmail.com).

$0022-5223 / \$ 36.00$

Copyright (c) 2015 by The American Association for Thoracic Surgery

http://dx.doi.org/10.1016/j.jtcvs.2015.07.003
}

A number of large-scale studies have demonstrated that $\beta$ blockers improve the survival of patients with cardiac failure and myocardial infarction as a result of their sympathoinhibitory and cardioprotective effects. ${ }^{1,2}$ Most of the previous studies have investigated oral $\beta$-blockers, however, and data on injectable $\beta$-blockers are inadequate. In addition, none of the studies of $\beta$-blockers were actually conducted in patients undergoing cardiac surgery. The role of $\beta$ blockers is currently attracting attention because cardiac surgery provokes a pathologic state of neurohumoral 


\section{Abbreviations and Acronyms \\ $\mathrm{POAF}=$ postoperative atrial fibrillation \\ $\mathrm{ACCF}=$ American College of Cardiology \\ Foundation \\ $\begin{array}{ll}\mathrm{AHA} & =\text { American Heart Association } \\ \mathrm{HR} & =\text { heart rate }\end{array}$ \\ PLATON $=$ Prevention of Atrial Fibrillation After \\ Cardiac Surgery With Landiolol \\ Hydrochloride for Left Ventricular \\ Dysfunction [trial] \\ ICU = intensive care unit \\ hs-CRP = high-sensitivity C-reactive protein \\ $\mathrm{BNP}=$ brain natriuretic peptide}

hyperactivity, including sympathetic overactivity. It is particularly important to prevent atrial fibrillation after cardiac surgery, because atrial fibrillation not only influences cardiac events and cerebral complications in the early postoperative period but also affects the long-term prognosis. Postoperative atrial fibrillation (POAF) is a common complication of cardiac surgery, and its incidence has been variously reported as $16 \%$ to $85 \% .^{3-7}$ POAF often occurs in the early period after surgery and has an influence on stroke and early postoperative cardiac events, as well as on the long-term prognosis, ${ }^{8}$ so prevention is important.

For the prevention of POAF, amiodarone and oral $\beta$-blockers are recommended in the American College of Cardiology Foundation (ACCF) and American Heart Association (AHA) guidelines. $^{9}$ Although the prophylactic administration of oral $\beta$-blockers is recommended, there have been few prospective studies on injectable $\beta$-blockers, and their efficacy has not been demonstrated, probably because the injectable drugs are more likely to cause hypotension or cardiac failure through their negative inotropic effect. $^{10,11}$

Landiolol hydrochloride (INN landiolol) was developed in Japan. Landiolol shows high $\beta_{1}$ selectivity, with its cardioselectivity $\left(\beta_{1} / \beta_{2}\right.$-receptor activation) being reported to be 8 times that of esmolol and 375 times that of propranolol. In addition, it has an extremely short half-life of approximately 4 minutes. When landiolol and esmolol were compared, both drugs caused a dose-dependent decrease of heart rate (HR), and esmolol also caused a marked decrease of blood pressure, whereas landiolol showed less effect on blood pressure because it has a weaker negative inotropic effect and a stronger chronotropic effect than esmolol. ${ }^{12-15}$

Because of these characteristics, we considered that landiolol was the most appropriate injectable $\beta$-blocker for patients with unstable postoperative hemodynamics, and we have previously conducted 2 prospective studies (the
Prevention of Atrial Fibrillation in Patients Having Cardiac Surgery with Landiolol Hydrochloride for Coronary Artery Bypass Grafting (PASCAL) and Effect of Beta-Blockers for Prophylaxis of Atrial Fibrillation after Coronary Artery Bypass Grafting with Intravenous Landiolol Hydrochloride and Oral Bisoprolol Administration (BABYLON) trials) on the use of landiolol during cardiac surgery. Both studies provided evidence that landiolol prevents POAF, presumably because it has anti-ischemic, anti-inflammatory, and antioxidant effects in addition to the standard actions of a $\beta$-blocker. ${ }^{16,17}$ A prospective study has not been performed to assess the safety and efficacy of landiolol for preventing POAF in patients with left ventricular dysfunction, however, so we conducted this clinical trial to determine the safety and efficacy of landiolol for prevention of POAF in patients with left ventricular dysfunction undergoing cardiac surgery (Prevention of Atrial Fibrillation After Cardiac Surgery With Landiolol Hydrochloride for Left Ventricular Dysfunction [PLATON] trial.

\section{MATERIALS AND METHODS \\ Study Protocol}

The PLATON trial was a randomized, open-label study. It was conducted after obtaining approval from the ethics committee of Nihon University School of Medicine Itabashi Hospital. Informed consent was provided by each patient after they received an explanation about this study. This study was registered with the University Hospital Medical Information Network (study ID: UMIN000002160).

The subjects were patients who underwent cardiac surgery under cardiopulmonary bypass and had left ventricular dysfunction (left ventricular ejection fraction $\leq 35 \%$ on preoperative left ventricular angiography or echocardiography before surgery). We excluded patients with sinus bradycardia (resting HR $\leq 50$ beats/min), second- or third-degree atrioventricular block, clinical hypothyroidism or hyperthyroidism, history of arrhythmia, surgery with circulatory arrest or left ventriculotomy, and planned off-pump surgery. Because the target population of this study, cardiac surgical patients with left ventricular dysfunction, is relatively small, we estimated that the feasible number of patients for recruitment was 30 per group. In our 2 previous studies, the incidences of POAF were approximately $35 \%$ in the placebo group and approximately $10 \%$ in the landiolol group. ${ }^{16,17}$ The incidence of POAF was therefore estimated to be $35 \%$ in the control group, and it was assumed that it would be decreased to $10 \%$ by treatment with landiolol. With 30 patients per group and the level of significance set at $5 \%$, the power of detection was calculated to be $78 \%$, suggesting that a sample size of 60 was sufficient for this study.

The patients were randomly assigned to 2 groups (a landiolol group and a control group of patients who did not receive landiolol) by the lottery method before surgery. We asked medical staff who were not involved in the surgery or postoperative management to choose an envelope containing the group assignment (landiolol group or control group) when the patient entered the operating room. Physicians and nurses involved in the surgery or postoperative management were not blinded, but the patients were blinded to the treatment assigned, as were the researchers who compiled the results.

In the landiolol group, infusion of landiolol hydrochloride (Ono Pharmaceutical Co, Ltd, Osaka, Japan) was started at a rate of $2 \mu \mathrm{g} /$ $\mathrm{kg} / \mathrm{min}$ at the time of weaning from cardiopulmonary bypass and 
was continued for at least 2 days. An oral $\beta$-blocker was administered from the day after oral intake was started. If an oral $\beta$-blocker was commenced on postoperative day 3 , the landiolol infusion rate was decreased to $1 \mu \mathrm{g} / \mathrm{kg} / \mathrm{min}$, and then infusion was discontinued after 1 hour. If an oral $\beta$-blocker was not commenced on postoperative day 3 , the infusion rate of landiolol was decreased to $1 \mu \mathrm{g} / \mathrm{kg} / \mathrm{min}$ when the oral $\beta$-blocker was actually administered, and then infusion was discontinued after 1 hour. The maximum duration of landiolol infusion was 5 days.

If the HR was 90 beats/min or less, right atrial pacing was performed at 90 beats/min for 48 hours. Continuous electrocardiographic monitoring was performed for 1 week after surgery. In this study, POAF was defined as atrial fibrillation that continued for at least 5 minutes or atrial fibrillation that had a negative influence on hemodynamics and required treatment; transient atrial fibrillation was excluded.

The primary end point was the occurrence or nonoccurrence of POAF during the initial 1-week period after surgery. The secondary end points were as follows: (1) operative mortality and complications; (2) hemodynamics, as assessed by systolic blood pressure, diastolic blood pressure, and HR (in patients on pacing, the pacemaker was stopped before measurement); (3) creatine kinase MB isozyme on returning to the intensive care unit (ICU), 3 hours after returning, and on postoperative days 1,2 , and 3 , and troponin-I and human heart fatty acid-binding protein on returning to the ICU and on postoperative days 1 and 3 as indices of ischemia-reperfusion injury; (4) high sensitivity C-reactive protein (hs-CRP) before surgery, on returning to ICU, and on postoperative days 1,3 , and 7 as an index of inflammation; (5) brain natriuretic peptide (BNP) before surgery, on return to the ICU, and on postoperative days 1 and 3; and (6) the lengths of ICU and hospital stays.

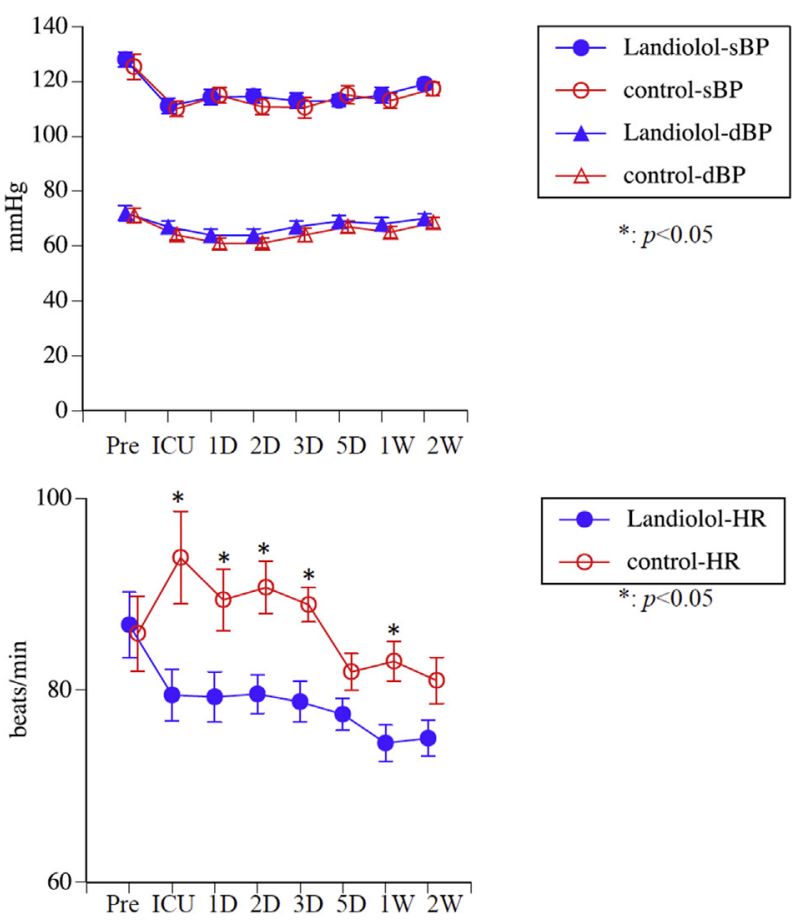

FIGURE 1. Changes in systolic blood pressure $(s B P)$ and diastolic blood pressure $(d B P)$ and in heart rate $(H R)$. Pre, Preoperative; ICU, intensive care unit; $D$, postoperative day; $W$, postoperative week.

\section{Statistical Analysis}

The Student $t$ test and Fisher exact test were used to assess the differences of continuous variables with a normal distribution, which are reported as mean $\pm \mathrm{SD}$, whereas the Mann-Whitney test was used for nonparametric data. Hemodynamic data are reported as mean $\pm \mathrm{SE}$, and differences were analyzed by repeated measures analysis of variance with a post hoc Scheffé test. The data shown in Figures 1 and 2 were subjected to logarithmic transformation before analysis because of an extremely skewed distribution. Repeated measures analysis of variance was performed with the patients as the random effect and time as the fixed effect, and the results were back-transformed (expressed as the geometric mean and $95 \%$ confidence interval).

\section{RESULTS}

\section{Patient Enrollment}

The 60 patients enrolled in the PLATON trial were randomly allocated to 2 groups, with 30 being assigned to the landiolol group and 30 to the control group. Intravenous infusion was not discontinued for hypotension, severe bradycardia, or atrioventricular block in either group, and all patients completed the study. Atrial pacing was performed in 11 patients from the landiolol group and 9 patients from the control group, with no significant difference being observed $(P=.785)$.

\section{Baseline Characteristics}

Preoperative patient characteristics showed no significant differences between the 2 groups (Table 1).

\section{Postoperative Results}

Table 2 shows the details of operative death, complications, surgical procedures, aortic crossclamp time, extracorporeal circulation time, postoperative respiratory support time, and postoperative oral medications. No significant differences were observed between the landiolol group and the control group. There were no deaths in the landiolol group, whereas 2 deaths occurred in the control group. One patient had myocardial infarction develop immediately after surgery. On postoperative day 4 , the onset of tachycardiac atrial fibrillation and ventricular tachycardia was managed with electrical cardioversion and intra-aortic balloon pumping. The arrhythmia became resistant to treatment, however, and the patient died on postoperative day 13. The other patient had atrial fibrillation develop on postoperative day 7 and had a cerebral infarction on postoperative day 10 . This patient died on postoperative day 22 without recovering consciousness. All other patients were discharged from the hospital. The hospital stay was significantly shorter in the landiolol group (median, 12.0 days; minimum, 8 days; maximum, 37 days; and interquartile range, 10.0-17.0 days) than in the control group (median, 14.0 days; minimum, 8 days; maximum, 54 days; and interquartile range, 11.0-22.5 days; $P=.029$ ). 

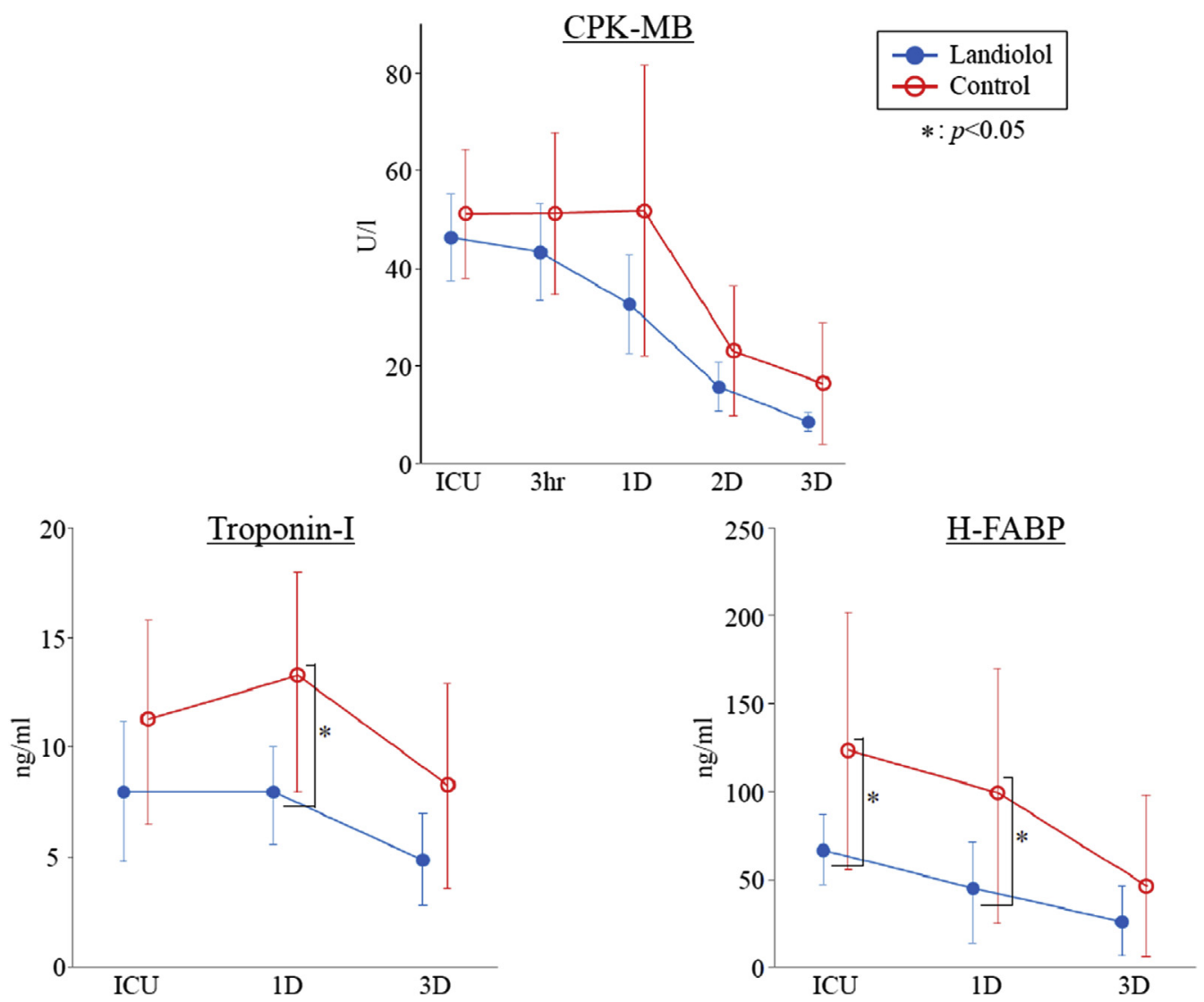

FIGURE 2. Changes in the ischemic biomarkers creatine kinase isoenzyme MB $(C P K-M B)$, troponin I, and human heart fatty acid-binding protein $(H$ $F A B P)$. ICU, Intensive care unit; $D$, postoperative day.

In the landiolol group, the duration of landiolol infusion was $2.67 \pm 0.82$ days ( 2 days in 21 patients, 3 days in 5,4 days in 3 , and 5 days in 1; Table 2).

\section{Primary End Point}

The primary end point of POAF (Figure 3) occurred in 3 patients $(10 \%)$ from the landiolol group versus 12 patients $(40 \%)$ from the control group, with a significantly lower incidence in the landiolol group $(P=.002)$. Onset of POAF was at a mean of $3.00 \pm 2.65$ days after surgery in the landiolol group and $3.08 \pm 1.98$ days in the control group, showing no significant difference in timing $(P=.562)$. All the patients who had POAF develop and survived were restored to sinus rhythm at the time of discharge from hospital.

\section{Secondary End Points}

Hemodynamics. In the hemodynamic evaluation (Figure 1), no significant differences were observed between the 2 groups with respect to the preoperative HR, systolic blood pressure, and diastolic blood pressure (HR, $P=.58$; systolic blood pressure, $P=.453$; and diastolic blood pressure, $P=.322$ ). Although there was no significant difference in blood pressure postoperatively, the HR was significantly lower in the landiolol group (on returning to the ICU, $P=.012$; on postoperative day 1 , $P=.018$; on postoperative day $2, P=.002$; on postoperative day $3, P=.001$; and at the end of postoperative week $1, P=.003)$.

Ischemic biomarkers. In the evaluation of ischemic biomarkers (Figure 2), creatine kinase MB isozyme did not show a significant difference between the 2 groups (on returning to the ICU, $P=.545 ; 3$ hours after returning, $P=.415$; on postoperative day $1, P=.239$; on postoperative day $2, P=.309$; and on postoperative day 3 , $P=.219)$. On returning to ICU and on postoperative day 3 , there was no significant difference in troponin-I between the 2 groups; however troponin-I was significantly lower in the landiolol group on postoperative day 1 $(P=.022)$. On returning to the ICU and on postoperative day 1 , heart fatty acid-binding protein was significantly lower in the landiolol group than in the control group $(P=.012$ and $P=.437$, respectively).

High-sensitivity $\mathrm{C}$-reactive protein. In the evaluation of hs-CRP level (Figure 4), no significant difference was observed between the landiolol group and the 
TABLE 1. Clinical characteristics of the landiolol and control groups

\begin{tabular}{lccr}
\hline & $\begin{array}{c}\text { Landiolol group } \\
(\mathbf{n}=\mathbf{3 0})\end{array}$ & $\begin{array}{c}\text { Control group } \\
(\mathbf{n}=\mathbf{3 0})\end{array}$ & $\begin{array}{c}\boldsymbol{P} \\
\text { value }\end{array}$ \\
\hline Age (y) & $64.8 \pm 9.6$ & $68.3 \pm 9.4$ & .842 \\
Age group & & & \\
$>80$ y & 4 & 2 & .389 \\
$>75$ y & 5 & 5 & $>.999$ \\
$>70$ y & 8 & 10 & .573 \\
Sex (male/female) & $26: 4$ & $24: 6$ & .488 \\
Emergency surgery & 5 & 8 & .347 \\
Reoperation & 1 & 0 & .313 \\
Risk factors & & & \\
Diabetes mellitus & 16 & 13 & .438 \\
Hypertension & 23 & 19 & .260 \\
Hyperlipidemia & 12 & 17 & .197 \\
Obesity & 6 & 3 & .278 \\
Smoking & 16 & 14 & .606 \\
COPD & 0 & 0 & $>.999$ \\
Cerebrovascular disease & 2 & 4 & .389 \\
Chronic renal failure & 13 & 19 & .121 \\
Hemodialysis & 6 & 8 & .542 \\
Oral medications & & & \\
ARB & 12 & 16 & .091 \\
ACE-I & 9 & 5 & .222 \\
Calcium antagonist & 9 & 7 & .559 \\
$\beta$-Blocker & 12 & .301 \\
Aldosterone blocker & $98.6 \pm 7.3$ & $28.6 \pm 6.0$ & .989 \\
Statin & & 559 \\
Ejection fraction (\%) & & & \\
\hline
\end{tabular}

Data represent number and percentage or as mean $\pm \mathrm{SD}$. COPD, Chronic obstructive pulmonary disease; $A R B$, angiotensin II receptor blocker; $A C E-I$, angiotensinconverting enzyme inhibitor.

control group with respect to the preoperative level $(P=.665)$; however, the hs-CRP was significantly lower in the landiolol group during postoperative week $2(P=.045)$.

Brain natriuretic peptide. Although there was no significant difference in preoperative BNP between the 2 groups $(P=.983)$, BNP was significantly lower in the landiolol group than in the control group from postoperative day 1 to postoperative month 1 (postoperative day $1, P<.001$; postoperative day $3, P<.001$; postoperative week 1 , $P=.006$; postoperative week $2, P=.011$; and postoperative month $1, P=.024$ ).

ICU and hospital stays. Whereas there was no significant difference in ICU stay between the landiolol group and the control group $(4.13 \pm 2.39$ days vs $4.30 \pm 2.94$ days; $P=.415)$, the hospital stay was significantly shorter in the landiolol group than in the control group $(P=.029)$.

\section{DISCUSSION}

Landiolol is an injectable $\beta$-blocker that has a very short half-life of 4 minutes, suggesting that low-dose infusion can be performed safely with little influence on
TABLE 2. Surgical procedure data and postoperative data of the landiolol and control groups

\begin{tabular}{|c|c|c|c|}
\hline & $\begin{array}{l}\text { Landiolol group } \\
\quad(\mathbf{n}=\mathbf{3 0})\end{array}$ & $\begin{array}{l}\text { Control group } \\
(\mathbf{n}=\mathbf{3 0})\end{array}$ & $\begin{array}{c}P \\
\text { value }\end{array}$ \\
\hline In-hospital death & 0 & 2 & .150 \\
\hline PMI & 0 & 1 & \\
\hline Cerebral infarction & 0 & 1 & \\
\hline Complications & 1 & 4 & .161 \\
\hline Bleeding & 1 & 0 & \\
\hline PMI & 0 & 1 & \\
\hline Cerebral infarction & 0 & 1 & \\
\hline $\begin{array}{l}\text { Complete AV block } \\
\text { (PM implantation) }\end{array}$ & 0 & 1 & \\
\hline Skin infection & 0 & 1 & \\
\hline Surgical procedure & & & .683 \\
\hline CABG & 23 & 23 & \\
\hline CABG and AVR & 0 & 1 & \\
\hline CABG and MVR & 1 & 0 & \\
\hline AVR & 4 & 5 & \\
\hline MVR & 1 & 0 & \\
\hline DVR & 1 & 1 & \\
\hline ACCT (min) & $62.2 \pm 63.9$ & $64.9 \pm 37.9$ & .340 \\
\hline ECCT (min) & $132.6 \pm 67.9$ & $135.5 \pm 41.3$ & .655 \\
\hline Ventilation (h) & $18.5 \pm 20.9$ & $24.9 \pm 29.3$ & .655 \\
\hline Landiolol use (d) & $2.67 \pm 0.82$ & - & - \\
\hline ICU stay (d) & $4.13 \pm 2.39$ & $4.30 \pm 2.94$ & .415 \\
\hline Hospital stay (d) & $14.6 \pm 6.4$ & $19.4 \pm 11.6$ & .019 \\
\hline \multicolumn{4}{|c|}{ Postoperative oral medications } \\
\hline ARB & 9 & 9 & $>.999$ \\
\hline ACE-I & 4 & 1 & .161 \\
\hline Calcium antagonist & 5 & 4 & .718 \\
\hline$\beta$-Blocker & 25 & 24 & .739 \\
\hline Aldosterone blocker & 22 & 23 & .766 \\
\hline Statin & 17 & 15 & .605 \\
\hline
\end{tabular}

Data represent number and percentage or as mean \pm SD. PMI, Perioperative myocardial infarction; $A V$, atrioventricular; $P M$, pacemaker; $C A B G$, coronary artery bypass grafting; $A V R$, aortic valve replacement; $M V R$, mitral valve replacement; $D V R$, double valve replacement; $A C C T$, aortic crossclamp time; $E C C T$, extracorporeal circulation time; $I C U$, intensive care unit; $A R B$, angiotensin II receptor blocker; $A C E-I$, angiotensin-converting enzyme inhibitor.

hemodynamics. In fact, our previous 2 prospective studies demonstrated that low-dose landiolol has no influence on hemodynamics, including no hypotension or bradycardia, in patients undergoing coronary artery bypass grafting.

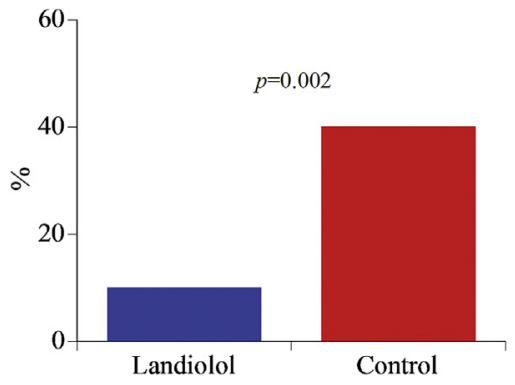

FIGURE 3. Occurrence of postoperative atrial fibrillation. 

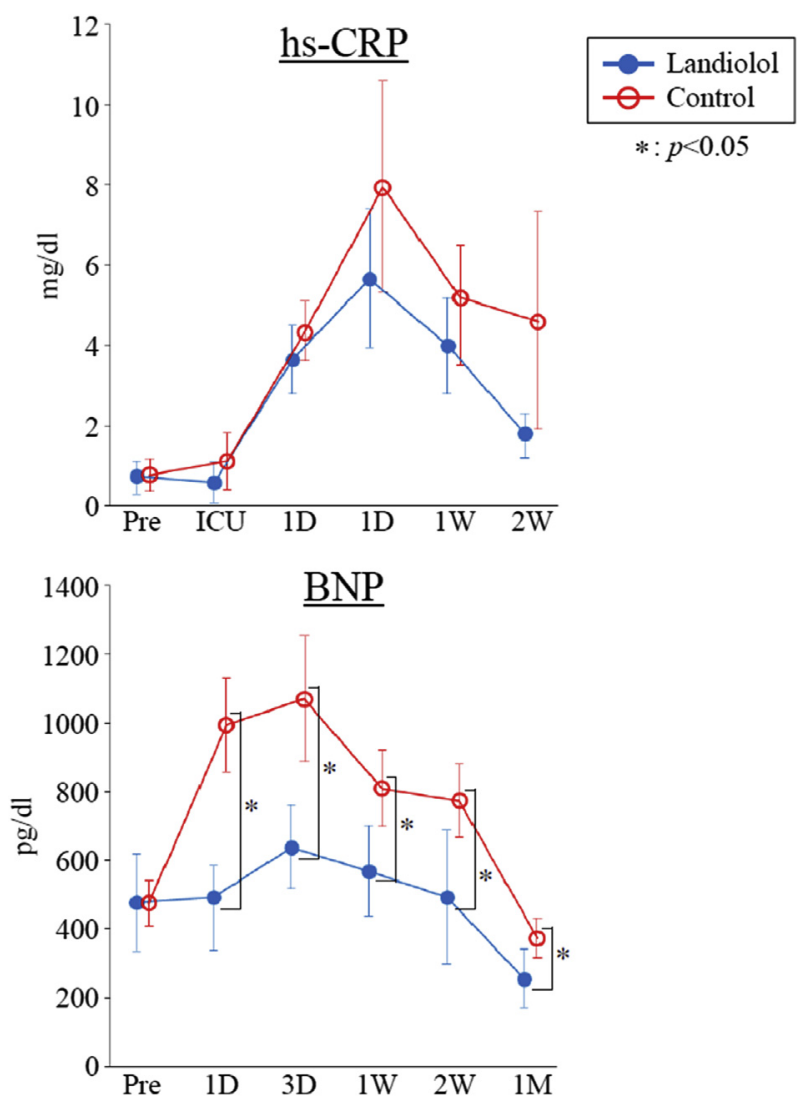

FIGURE 4. Changes in highly sensitive C-reactive protein $(h s-C R P)$ and brain natriuretic peptide $(B N P)$. Pre, Preoperative; $I C U$, intensive care unit; $D$, postoperative day; $W$, postoperative week, $M$, postoperative month.

There have been reports that atrial pacing prevents occurrence of POAF, ${ }^{18}$ or that there is no difference between $\beta$ blockers and amiodarone. ${ }^{19,20}$ This issue is not covered by the current ACCF and AHA guideline, ${ }^{9}$ and no consensus has been reached. Accordingly, we conducted this prospective study to investigate the safety and efficacy of landiolol in patients with left ventricular dysfunction undergoing cardiac surgery. We found that low-dose infusion of landiolol did not compromise hemodynamics and that the occurrence of bradycardia during landiolol infusion immediately after surgery could be safely managed by atrial pacing. There were no patients with severe bradycardia or atrioventricular block, and low-dose landiolol safely prevented POAF in cardiac surgical patients with ventricular dysfunction. According to the 2011 ACCF and AHA guideline, "The effectiveness of preoperative beta blockers in reducing in-hospital mortality rate in patients with left ventricular ejection fraction less than $30 \%$ is uncertain" (class IIb). ${ }^{9}$ It is therefore considered noteworthy that this study demonstrated the safety and efficacy of perioperative intravenous infusion of landiolol for preventing POAF in patients with left ventricular dysfunction. The ACCF and AHA guideline states that oral $\beta$-blockers are recommended for prophylaxis of POAF, but a retrospective review of the Society of Thoracic Surgeons database ( $>50,000$ patients) found a significantly higher incidence of POAF in patients given oral $\beta$-blockers within 24 hours before surgery than in patients not treated with $\beta$-blockers. ${ }^{21}$ This suggests that administering oral $\beta$-blockers immediately before surgery may not prevent POAF. In contrast, perioperative intravenous infusion of landiolol was safe and effective for preventing POAF in this study and our previous studies. ${ }^{16,17}$ Patients with left ventricular dysfunction (the subjects of this study) are often treated with oral $\beta$-blockers; if they are not already receiving oral $\beta$-blocker therapy, however, we consider that an intravenous $\beta$-blocker should be infused perioperatively irrespective of cardiac function, rather than starting an oral agent immediately before surgery.

The reduction of HR in the landiolol group during the early postoperative period demonstrated the sympathoinhibitory effect of this drug, which may prevent an early postoperative increase of BNP and reduce the cardiac workload, as well as having a cardioprotective effect. These points should be investigated in detail in the future by cardiac catheterization, echocardiography, magnetic resonance imaging, and other modalities.

With regard to biomarkers of ischemia, creatine kinase MB isozyme showed no difference between the 2 groups, but troponin-I (postoperative day 1) and heart fatty acid-binding protein (postoperative days 1 and 3) were significantly lower in the landiolol group than in the control group. Because of the small number of subjects in this study, we could not assess the relationships between these markers and operative death or complications. Our results suggest the possibility that landiolol prevents ischemia, however, which warrants further investigation.

The inflammatory marker hs-CRP was found to be significantly lower in the landiolol group in postoperative week 2 alone. Inflammatory markers are generally increased by operative stress, whereas the effect of $\beta$-blockers on inflammation in postoperative week 2 is unknown. In our previous study, there was no difference in hs-CRP between patients with and without $\beta$-blocker therapy, but pentraxin-3 (a more sensitive inflammatory marker than hs-CRP) was significantly lower in the $\beta$-blocker group during the early postoperative period. ${ }^{17}$ This suggests that the antiinflammatory effects of $\beta$-blockers should be investigated further in the future.

There have been some reports about the efficacy of landiolol for cardiac surgery, ${ }^{16,17,22-24}$ but no randomized, controlled trials have previously been performed in patients with left ventricular dysfunction undergoing cardiac surgery. Ito and colleagues ${ }^{25}$ 
performed the only previous study of landiolol in cardiac surgical patients with left ventricular dysfunction. It was a retrospective study, however, and the groups were not well balanced, with a significantly larger number of patients undergoing coronary artery bypass grafting alone in the landiolol group and a significantly larger number of patients undergoing coronary artery bypass grafting combined with valve surgery in the control group. They reported a significant decrease in HR postoperatively in the landiolol group, with no change of blood pressure and a significantly lower frequency of POAF in the landiolol group relative to the control group.

Unlike landiolol, most intravenous $\beta$-blockers can cause hypotension as a result of their cardiodepressant effect. ${ }^{10,26}$ Esmolol is another ultrashort-acting $\beta$ blocker (half-life of 9 minutes) that is used in Europe and the United States. Gray and colleagues ${ }^{27}$ used esmolol to treat 24 patients with postoperative tachyarrhythmia and achieved a decrease in HR by $15 \%$ or more in all cases. Asymptomatic hypotension occurred in 13 patients, however, and administration was discontinued in 2 patients. Injectable $\beta$-blockers have a faster onset of action than oral drugs. Halonen and coworkers ${ }^{28}$ reported that intravenous administration of metoprolol was more effective than oral administration for preventing atrial fibrillation, with the incidence of atrial fibrillation being $28.1 \%$ in the oral group versus $16.8 \%$ in the intravenous group. In contrast, Maniar and associates ${ }^{10}$ compared the prophylactic effects on atrial fibrillation of intravenous esmolol with those of oral $\beta$-blockers and found no difference of POAF between the 2 groups, although hypotension was significantly more frequent in the esmolol group. A large-scale study by Chen and colleagues ${ }^{11}$ comparing early intravenous administration of metoprolol and oral administration in 45,852 patients with acute myocardial infarction showed that the incidence of fresh nonfatal myocardial infarction and ventricular fibrillation was lower in the intravenous group than in the oral group. Chen and colleagues ${ }^{11}$ recommended that intravenous metoprolol be used only when hemodynamics were stable, however, because there was a high incidence of cardiogenic shock on the day of infarction in the intravenous metoprolol group. Because injectable $\beta$-blockers with a prolonged duration of action can severely compromise hemodynamics by causing hypotension or bradycardia, the use of these drugs during cardiac surgery is generally not recommended.

Previous prospective studies have demonstrated a preventive effect of landiolol hydrochloride on POAF after cardiac surgery. ${ }^{16,17,22-24}$ Landiolol and diltiazem were compared for the treatment of tachyarrhythmia after cardiac surgery in the Japan Landiolol Kick-Off of
Novel Investigation for Gold Standard Heart (JLKNIGHT) study, revealing that sinus rhythm was restored at a significantly higher rate with a lower incidence of hypotension and bradycardia in the landiolol group, indicating that landiolol was more effective and safer. ${ }^{22}$ Because the efficacy of oral $\beta$ blockers has been established for cardiac failure and acute myocardial infarction, a Japanese multicenter study was performed to compare landiolol and digoxin in patients with cardiac dysfunction and atrial tachyarrhythmia. It was found that the rate of achieving the primary end point (HR of 110 beats/min or lower and reduction of HR by $20 \%$ or more at 2 hours after starting administration) was significantly higher in the landiolol group $(48 \%)$ than in the digoxin group $(13.9 \%)$, with a lower incidence of adverse reactions including hypotension in the former group. ${ }^{29}$ Because the efficacy and safety of landiolol hydrochloride were demonstrated in patients with cardiac dysfunction by that study, the use of landiolol is expected to increase in the field of cardiovascular medicine.

In conclusion, POAF was significantly less frequent after cardiac surgery in the landiolol group than in the control group $(10 \%$ vs $40 \%)$, and intravenous landiolol hydrochloride prevented POAF in patients who had left ventricular dysfunction without causing hypotension or bradycardia. Accordingly, landiolol may be a promising addition to the perioperative management of patients with left ventricular dysfunction undergoing cardiac surgery.

\section{Limitations}

This trial was not blinded, because intravenous $\beta$ blockers have the potential to cause severe complications such as hypotension during the perioperative period when hemodynamic instability is likely and also because landiolol has not been investigated previously in cardiac surgical patients with left ventricular dysfunction. Now that the safety of low-dose landiolol infusion has been demonstrated by the present study, blinded investigations could be conducted in the future.

\section{Conflict of Interest Statement}

A.S. has received lecture fees from Ono Pharmaceutical Co, Ltd, Dai-ichi Sankyo Co, Ltd, and Teijin Co, Ltd. All other authors have nothing to disclose with regard to commercial support.

\section{References}

1. Beta-Blocker Evaluation of Survival Trial Investigators. A trial of the betablocker bucindolol in patients with advanced chronic heart failure. $N$ Engl $J$ Med. 2001;344:1659-67. 
2. Dargie HJ. Effect of carvedilol on outcome after myocardial infarction in patients with left-ventricular dysfunction: the CAPRICORN randomised trial. Lancet. 2001;357:1385-90.

3. Villareal RP, Hariharan R, Liu BC, Kar B, Lee VV, Elayda M, et al. Postoperative atrial fibrillation and mortality after coronary atrial bypass surgery. J Am Coll Cardiol. 2004;43:742-8.

4. Sezai A, Shiono M. The role of $\beta$-blockers in cardiac perioperative management. Ann Thorac Cadiovasc Surg. 2014;20:261-6.

5. Sezai A, Shiono M. Atrial fibrillation after cardiac surgery. Circ J. 2013;77: 2244-5.

6. Budeus M, Hennersdorf M, Perings S, Röhlen S, Schnitzler S, Felix O, et al. Amiodarone prophylaxis for atrial fibrillation of high-risk patients after coronary bypass grafting: a prospective, double-blind, placebo-controlled, randomized study. Eur Heart J. 2006;27:1584-91.

7. Sezai A, Hata M, Niino T, Kasamaki Y, Nakai T, Hirayama A, et al. Study of the factors related to atrial fibrillation after coronary artery bypass grafting: a search for a marker to predict the occurrence of atrial fibrillation before surgical intervention. J Thorac Cardiovasc Surg. 2009;137:895-900.

8. Mariscalco G, Klersy C, Zanobini M, Banach M, Ferrarese S, Borsani P, et al. Atrial fibrillation after isolated coronary surgery affects late survival. Circulation. 2008;118:1612-8.

9. Hillis LD, Amith PK, Anderson JL, Bittl JA, Bridges CR, Byrne JG, et al. 2011 ACCF/AHA/guideline for coronary artery bypass grafting. a report of the American College of Cardiology Foundation/American Heart Association Task Force on Practice Guidelines. Circulation. 2011;124:e652-735.

10. Maniar PB, Balcetyte-Harris N, Tamis JE, Steinberg JS. Intravenous versus oral beta-blockers for prevention of post-CABG atrial fibrillation in high-risk patients identified by signal-averaged ECG: lessons of a pilot study. Card Electrophysiol Rev. 2003;7:158-61.

11. Chen ZM, Pan HC, Chen YP, Peto R, Collins R, Jiang LX, et al. Early intravenous then oral metoprolol in 45,852 patients with acute myocardial infarction: randomised placebo-controlled trial. Lancet. 2005;366:1622-32.

12. Plosker GL. Landiolol: a review of its use in intraoperative and postoperative tachyarrhythmias. Drugs. 2013;73:959-77. Erratum in: Drugs. 2013;73:1255.

13. Iguchi S, Iwamura H, Nishizaki M, Hayashi A, Senokuchi K, Kobayashi K, et al. Development of a highly cardioselective ultra short-acting $\beta$-blocker, ONO1101. Chem Pharm Bull (Tokyo). 1992;40:1462-9.

14. Sugiyama A, Takahara A, Hashimoto K. Electrophysiologic, cardiohemodynamic, and $\beta$-blocking actions of a new ultra-short-acting $\beta$-blocker, ONO1101 , assessed by the in vivo canine model in comparison with esmolol. J Cardiovasc Pharmacol. 1999;34:70-7.

15. Sasao J, Tarver SD, Kindscher JD, Taneyama C, Benson KT, Goto H. In rabbits, landiolol, a new ultra-short-acting $\beta$-blocker, exerts a more potent negative chronotropic effect and less effect on blood pressure than esmolol. Can J Anesth. 2001;48:985-9.

16. Sezai A, Minami K, Nakai T, Hata M, Yoshitake I, Wakui S, et al. Landiolol hydrochloride for prevention of atrial fibrillation after coronary artery bypass grafting: new evidence from the PASCAL trial. J Thorac Cardiovasc Surg. 2011;141: 1478-87.

17. Sezai A, Nakai T, Hata M, Yoshitake I, Shiono M, Kunimoto S, et al. Feasibility of landiolol and bisoprolol for prevention of atrial fibrillation after cor- onary artery bypass grafting: a pilot study. J Thorac Cardiovasc Surg. 2012; 144:1241-8.

18. Greenberg MD, Katz NM, Iuliano S, Tempesta BJ, Solomon AJ. Atrial pacing for the prevention of atrial fibrillation after cardiovascular surgery. J Am Coll Cardiol. 2000;35:1416-22.

19. Maaroos M, Halonen J, Kiviniemi V, Hartikainen J, Hakala T. Intravenous metoprolol versus biatrial pacing in the prevention of atrial fibrillation after coronary artery bypass surgery: a prospective randomized open trial. Scand J Surg. 2012; 101:292-6.

20. Shea J, Sodhi G, Najam F, Solomon AJ. Does the addition of atrial pacing to amiodarone reduce the incidence of postoperative atrial fibrillation: a pilot study. J Thorac Cardiovasc Surg. 2014;148:1752-3.

21. Brinkman W, Herbert MA, O'Brien S, Filardo G, Prince S, Dewey T, et al. Preoperative $\beta$-blocker use in coronary artery bypass grafting surgery: national database analysis. JAMA Intern Med. 2014;174:1320-7.

22. Sakamoto A, Kitakaze M, Takamoto S, Namiki A, Kasanuki H, Hosoda S. JL-KNIGHT study group. Landiolol, an ultra-short-acting $\beta$-blocker, more effectively terminates atrial fibrillation than diltiazem after open heart surgery: prospective, multicenter, randomized, open-label study (JL-KNIGHT study). Circ J. 2012;76:1097-101.

23. Sakamoto A, Hamasaki T, Kitakaze M. Perioperative landiolol administration reduces atrial fibrillation after cardiac surgery: a meta-analysis of randomized controlled trials. Adv Ther. 2014;31:440-50.

24. Nagaoka E, Arai H, Tamura K, Makita S, Miyagi N. Prevention of atrial fibrillation with ultra-low dose landiolol after off-pump coronary artery bypass grafting. Ann Thorac Cardiovasc Surg. 2014;20:129-34.

25. Ito N, Tashiro T, Morishige N, Nishimi M, Hayashida Y, Minematsu N, et al. Safety and efficacy of an ultrashort-acting $\beta 1$-blocker on left ventricular dysfunction. Heart Surg Forum. 2012;15:E189-94.

26. Mooss AN, Wurdeman RL, Mohiuddin SM, Reyes AP, Sugimoto JT, Scott W, et al. Esmolol versus diltiazem in the treatment of postoperative atrial fibrillation/atrial flutter after open heart surgery. Am Heart J. 2000; 140:176-80.

27. Gray RJ, Bateman TM, Czer LC, Conklin CM, Matloff JM. Esmolol: A new ultrashort-acting beta-adrenergic blocking agent for rapid control of heart rate in postoperative supraventricular tachyarrhythmias. J Am Coll Cardiol. 1985;5: 1451-6.

28. Halonen J, Hakala T, Auvinen T, Karjalainen J, Turpeinen A, Uusaro A, et al. Intravenous administration of metoprolol is more effective than oral administration in the prevention of atrial fibrillation after cardiac surgery. Circulation. 2006; 114(1 Suppl):I1-4.

29. Nagai R, Kinugawa K, Inoue H, Atarashi H, Seino Y, Yamashita T, et al. Urgent management of rapid heart rate in patients with atrial fibrillation/ flutter and left ventricular dysfunction: comparison of the ultra-shortacting $\beta 1$-selective blocker landiolol with digoxin (J-Land Study). Circ J. 2013;77:908-13.

Key Words: beta blocker, atrial fibrillation, cardiac surgery, left ventricular dysfunction 